\title{
Enhanced Damage Resistance and Novel Defect Structure of CrFeCoNi under In Situ Electron Irradiation
}

\author{
Mo-Rigen He, ${ }^{a}$ Shuai Wang, ${ }^{a}$ Ke Jin,,${ }^{\mathrm{b}}$ Hongbin Bei, ${ }^{\mathrm{b}}$ Kazuhiro Yasuda, ${ }^{\mathrm{c}}$ Syo Matsumura, ${ }^{\mathrm{c}}$ \\ Kenji Higashida, ${ }^{\mathrm{d}}$ Ian M. Robertson ${ }^{\mathrm{a}, *}$ \\ ${ }^{a}$ Department of Engineering Physics, University of Wisconsin-Madison, Madison, WI \\ 53706, USA \\ ${ }^{\mathrm{b}}$ Materials Science and Technology Division, Oak Ridge National Laboratory, Oak Ridge, \\ TN 37831, USA \\ ${ }^{\mathrm{c}}$ Ultramicroscopy Research Center and Department of Applied Quantum Physics and \\ Nuclear Engineering, Kyushu University, Fukuoka 819-0395, Japan \\ ${ }^{\mathrm{d}}$ Department of Materials Science and Engineering, Kyushu University, Fukuoka \\ 819-0395, Japan \\ *E-mail address: irobertson@wisc.edu (I. M. Robertson).
}

\begin{abstract}
Defect production and growth in $\mathrm{CrFeCoNi}$, a single-phase concentrated solid solution alloy, is characterized using in situ electron irradiation inside a transmission electron microscope operated at 400 1250 kV and $400{ }^{\circ} \mathrm{C}$. All observed defects are interstitial-type, either elliptical Frank loops or polygonal (mostly rhombus) perfect loops. Both forms of loops in $\mathrm{CrFeCoNi}$ exhibit a sublinear power law of growth that is more than 40 times slower than the linear defect growth in pure Ni. This result shows how compositional complexity impacts the production of Frenkel pairs and the agglomeration of interstitials into loops, and, thus, enhances the radiation tolerance.
\end{abstract}

Keywords: concentrated solid solution alloys, radiation, defects, transmission electron microscopy. 
A common strategy to enhance the radiation tolerance of materials is to increase the density of sinks for the annihilation of radiation-induced defects [1-4]. On the other hand, it has been shown that defect production and growth behavior in several binary alloys, such as $\mathrm{Ni}_{1-x} \mathrm{Cr}_{x}$ [5] and $\mathrm{Ni}_{1-x} \mathrm{Cu}_{x}$ [6], can be moderated by the concentration $(x)$ of alloying elements. Along this line, single-phase concentrated solid solution alloys, a novel class of materials containing multiple (and often equiatomic) principal elements that are randomly arranged in a simple crystal lattice [7-10], open new prospects of controlling the early stages of energy dissipation and defect evolution due to their extreme compositional disorder and/or inherent lattice distortions [11-13]. For example, fewer and smaller extended defects (dislocation loops, etc.) were produced in $\mathrm{Ni}_{50} \mathrm{Co}_{50}$ and $\mathrm{Ni}_{50} \mathrm{Fe}_{50}$ than in $\mathrm{Ni}$ under ion irradiations [12]. This difference is attributed to the decrease of electron mean free path and thermal conductivity that prolong the lifetime of the thermal spike associated with a displacement cascade [12], as well as the increase of defect migration barriers [13]. A high phase stability was reported in nanocrystalline $\mathrm{ZrHfNb}$ [14] and $\mathrm{CrFeCoNiCu} \mathrm{[15]} \mathrm{alloys} \mathrm{subjected} \mathrm{to} \mathrm{electron} \mathrm{irradiation.} \mathrm{In} \mathrm{this} \mathrm{work,}$ we report the evolution and structure of defects produced in a $\mathrm{CrFeCoNi}$ alloy by in situ electron irradiation inside a transmission electron microscope (TEM), and demonstrate the enhanced damage resistance of these compositionally complex alloys under radiation conditions in which interstitial-vacancy pairs and not displacement cascades are generated.

The equiatomic $\mathrm{CrFeCoNi}$ alloy used in this study was synthesized as a single crystal and confirmed to be a single-phase solid solution with a face-centered cubic structure [16]. Disks with a diameter of $3 \mathrm{~mm}$ and a normal close to [001] direction were cut and thinned to electron-transparency by twin-jet electropolishing. In situ electron irradiations were performed inside a JEM-1300NEF high-voltage electron microscope (HVEM) at a nominal temperature of $400{ }^{\circ} \mathrm{C}$, with the beam energy varied between 400 and $1250 \mathrm{kV}$. The electron dose rate was kept constant at $\sim 5 \times 10^{18} \mathrm{e} \cdot \mathrm{cm}^{-2} \cdot \mathrm{s}^{-1}$ and all irradiations were 
conducted in areas with a thickness of $\sim 200 \mathrm{~nm}$, as assessed using the effective extinction distance and the number of thickness contours. Evolution of radiation-induced defects were recorded using a (g, 3.1g) weak-beam dark-field (WBDF) condition with $\mathbf{g}=(200)^{*}$. Post-mortem characterization of the defects in terms of their Burgers vectors (b), habit plane, and nature was performed using a Tecnai TF-30 TEM operating at $300 \mathrm{kV}$, by using the $\mathbf{g} \cdot \mathbf{b}$ invisibility criterion and the inside-outside contrast method [17].

The evolution of the defect structure in the CrFeCoNi alloy as irradiated by 1250,500 , and $400 \mathrm{kV}$ electron beam is summarized in Figure 1. Substantial defect production and growth were observed at $1250 \mathrm{kV}$ (Figure 1(a)), with elliptical faulted and polygonal perfect dislocation loops produced. The majority of loops nucleated within $1 \mathrm{~min}$ of irradiation, and the loop density saturated within 5 min. A one-to-one correspondence was found between the loops seen in Figure 1a(1) (5 min) and 1a(2) (30 min), despite the growth in loop sizes. The two forms of loops were produced simultaneously, in a number ratio close to 3 (faulted): 1 (perfect). No faulted loop was observed to transform to a perfect loop during its growth, and vice versa. Decreasing the beam energy to $500 \mathrm{kV}$ resulted in a decrease in both the density and size of loops (Figure 1(b)). Dislocation loops were still observed at $400 \mathrm{kV}$, although their production and growth rate was very low (Figure 1(c)). Observable damage was not produced within $2 \mathrm{~h}$ of irradiation, when the beam energy was reduced to $300 \mathrm{kV}$. These results suggest a displacement threshold energy $\left(E_{\mathrm{d}}\right)$ of $15 \sim 21 \mathrm{eV}$.

The density and mean size of loops is plotted in Figure 1(d), as a function of damage level (displacement per atom, dpa) that was calculated assuming $E_{\mathrm{d}}=15 \mathrm{eV}$ and using the scattering cross-section values in ref. [18]. Herein, the density includes both forms of loops, and the loop size is measured along the major axis that is always parallel with the (001) projected plane. The loop density saturated at a consistent damage level of $\sim 0.2 \mathrm{dpa}$, irrespective of electron beam energy. More intriguingly, the loop size versus damage level measured at 400 1250 kV collapsed onto a single curve, which can be fitted $\left(R^{2} \sim 0.99\right)$ 
by a power law: size $/ \mathrm{nm}=3.9 \times(\text { damage/dpa })^{0.69}$. Thus, we suggest that $E_{\mathrm{d}}$ along [001] direction of $\mathrm{CrFeCoNi}$ is around $15 \mathrm{eV}$, which is remarkably lower than $E_{\mathrm{d}}$ of pure $\mathrm{Ni}$ as evaluated previously [19, 20]: ${E_{\mathrm{d}}}^{[001]}=34 \sim 38 \mathrm{eV},{E_{\mathrm{d}}}^{[110]}=21 \sim 23 \mathrm{eV}$, and ${E_{\mathrm{d}}}^{[111]}=55 \sim 60$ $\mathrm{eV}$. This deviation can be rationalized in two ways. First, $E_{\mathrm{d}}^{[001]}$ of the $\mathrm{CrFeCoNi}$ alloy could be inherently lower than that of pure $\mathrm{Ni}$, seeing that $E_{\mathrm{d}}{ }^{[001]}$ of $\mathrm{Cr}$ and $\mathrm{Fe}$ (both have a body-centered cubic structure, though) is only $15 \sim 17 \mathrm{eV}[21,22]$, and $E_{\mathrm{d}}^{[001]}$ of Co (with a face-centered cubic structure) is $\sim 30 \mathrm{eV}$ [23], also lower than that of Ni. Second, there is a notable discrepancy in the values of $E_{\mathrm{d}}$ measured by HVEM experiments. For instance, dislocation loops were still observed in pure $\mathrm{Ni}$ irradiated by $440 \mathrm{kV}$ electron beam (even at room temperature) and along [001] direction, corresponding to an $E_{\mathrm{d}}{ }^{[001]}$ of $23 \mathrm{eV}$ [24]. $E_{\mathrm{d}}$ in solid solution alloys is complex and has been found to be affected by the local compositional order [20, 22].

Prior to quantification of the growth behavior of dislocation loops in $\mathrm{CrFeCoNi}$, the crystallography of these loops was determined. Figure 2 shows diffraction contrast analysis of a representative faulted loop. Figure 2(a) $\left(\mathbf{g}=(\overline{22} 0)^{*}\right)$ and $2(\mathrm{~b})\left(\mathbf{g}=(220)^{*}\right)$ show the changing width of the loop in WBDF images on reversing $\mathbf{g}$, with the "outside" contrast in Figure 2(a) corresponding to $(\mathbf{g} \cdot \mathbf{b}) s_{\mathbf{g}}>0$ (deviation vector $s_{\mathbf{g}}$ was positive). Moreover, the loop shows no contrast $(\mathbf{g} \cdot \mathbf{b}=0)$ in Figure 2(c) $\left(\mathbf{g}=(\overline{2} 20)^{*}\right)$ and 2(d) $(\mathbf{g}=$ $\left.(02 \overline{2})^{*}\right)$. Thus, b was identified to be $[\overline{1} \overline{1} \overline{1}] / 3$, corresponding to an interstitial-type Frank loop since $\mathbf{e} \cdot \mathbf{b}<0(\mathbf{e}=[001]$ or [011] was the incident direction of the electron beam). Figure 2(e) and 2(f) confirm the (111) habit plane and elliptical shape of this loop, with the major axis along [1 10$]$. Importantly, the unfaulting of interstitial-type Frank loops by absorbing supersaturated vacancies, which resulted in the "tongue-shaped" or multilayer faulted loops as commonly observed in pure $\mathrm{Ni}[24,25]$ irradiated under comparable conditions, was not observed in this alloy.

Figure 3 shows similar analysis of a representative perfect loop. Figure 3(a) $(\mathbf{g}=$ 
$\left.(\overline{2} 20)^{*}\right)$ and 3(b) $\left(\mathbf{g}=(220)^{*}\right)$ yielded "inside" and "outside" contrast, respectively, while the loop shows weak contrast, which is attributed to $\mathbf{g} \cdot(\mathbf{b} \times \mathbf{u}) \neq 0$ when $\mathbf{g} \cdot \mathbf{b}=0$, in Figure 3(c) $\left(\mathbf{g}=(\overline{1} 11)^{*}\right)$ and $3(\mathrm{~d})\left(\mathbf{g}=(111)^{*}\right)$. Thus, b was identified to be $[01 \overline{1}] / 2$ and the loop nature is also interstitial-type. Figure $3(\mathrm{e})$ shows the $(01 \overline{1})$ habit plane of this loop, making it a pure-edge loop, and Figure 3(f) determines the edges (u) of this rhombus loop to lie along [211] and [211], and the major axis along [100]. Only in quenched Al-based alloys have rhombus perfect loops with $\mathbf{b}=\langle 011\rangle / 2$ and $\mathbf{u}=\langle 211\rangle$ been reported previously [26]. These were assumed to evolve during the growth of vacancy-type Frank loops, which firstly unfaulted by absorption/nucleation of a Shockley partial dislocation [27], then reoriented from (111) to (110) habit plane by gliding along its Burgers vector [28]. However, this formation mechanism is at variance with the current observations in $\mathrm{CrFeCoNi}$, since the perfect loops in this alloy existed from the onset of visible damage formation, and unfaulting of Frank loops was not observed due to the low stacking fault energy [11]. Moreover, the perfect loops in $\mathrm{CrFeCoNi}$ were interstitial-type, which have not been reported to exist in electron irradiated Ni-based alloys.

Several individual dislocation loops in $\mathrm{CrFeCoNi}$ were monitored during in situ irradiation by $1250 \mathrm{kV}$ electron beam, and their growth behavior was quantified. Figure 4(a) and 4(b) demonstrate the growth of a Frank loop and a perfect loop, respectively, in a series of WBDF images captured at irradiation times of 2, 15, and $30 \mathrm{~min}$. Outlines of the loops are projected onto their habit planes to show the evolution of loop geometry. The growth of loop sizes (along major axis) are then plotted as a function of damage level in Figure 4(c), and can be fitted by a power law with the exponent (n) varying from 0.5 (for smaller loops) to 1.0 (for larger loops). No systematic difference was seen in the growth rates of Frank loops and perfect loops. On the other hand, Figure 4(d) shows that the aspect ratio of perfect loops was almost constant during growth, whereas the aspect ratio 
of Frank loops steadily increased with irradiation time. Such preferentially elongated Frank loops were observed in electron irradiated $\mathrm{Ni}$ [24] when the loops were large enough so that their growth along the minor axis, which was inclined to the free surfaces of the thin foils, was suppressed by the annihilation of point defects [29]. However, it is unclear if the two forms of loops form at different depths with respect to the free surface. Electron tomography techniques will be used in future work to determine the through-thickness distribution of these loops.

Finally, the loop growth during electron irradiation of pure Ni [24] is compared to that of $\mathrm{CrFeCoNi}$ alloy. As Figure 4(c) shows, a linear growth law $(n=1.0)$ of Frank loops was consistently observed in pure $\mathrm{Ni}$ above $400{ }^{\circ} \mathrm{C}[24,30]$. Loop growth models suggested that $n=1.0$ if surfaces serve as the dominating sinks for interstitials, while $n=$ 0.5 if recombination between vacancies and interstitials dominate [30]. These results support our expectation that the mobility of both vacancies and interstitials is reduced in this $\mathrm{CrFeCoNi}$ alloy, either due to the enhanced solute pinning [27] or the increased migration barrier of point defects [13], which promotes their recombination. In consequence, their agglomeration into dislocation loops was dramatically suppressed. Growth rates of $45 \sim 110 \mathrm{~nm} / \mathrm{dpa}$ (assuming $E_{\mathrm{d}}=15 \mathrm{eV}$ ) were measured for both forms of loops in $\mathrm{CrFeCoNi}$, while the growth rates of Frank loops in pure Ni were 2400 4300 $\mathrm{nm} / \mathrm{dpa}$ (assuming $E_{\mathrm{d}}=23 \mathrm{eV}$ ), manifesting an enhancement of radiation damage resistance by a factor of more than 40 solely due to the increase of compositional complexity. Such an effect was qualitatively insensitive to the values selected for $E_{\mathrm{d}}$, as a factor of $\sim 25$ was still obtained if $E_{\mathrm{d}}=20 \mathrm{eV}$ was used for all calculations. Therefore, compositional complexity decreases the survival of point defects produced by electron irradiation as well as the migration rate of interstitials and their agglomeration into loops.

In summary, evolution of radiation damage and defect structure in an equiatomic $\mathrm{CrFeCoNi}$ alloy is characterized using in situ electron irradiation. Concentrated alloying modulates the defect dynamics, such as displacement threshold energy, point defect 
migration energies, defect recombination efficiency, and formation mechanism of dislocation loops. Defect growth in $\mathrm{CrFeCoNi}$ is more than 40 times slower than in pure $\mathrm{Ni}$, demonstrating that compositional complexity can be used as a fundamentally new approach to the design of radiation-tolerant alloys.

\section{Acknowledgements}

The work was supported as part of Energy Dissipation to Defect Evolution (EDDE), an Energy Frontier Research Center funded by the U.S. Department of Energy, Office of Science. The electron microscopy with in situ electron irradiation was accomplished at Ultramicroscopy Research Center at Kyushu University, Japan with technical assistance from Dr. Tomokazu Yamamoto, as a project of the HVEM Collaborative Research Program sponsored by MEXT of the Japanese Government. Instrument support was also provided by Materials Research Science and Engineering Center (DMR-1121288) and Nanoscale Science and Engineering Center (DMR-0832760) at University of Wisconsin-Madison.

\section{References}

[1] X.M. Bai, A.F. Voter, R.G. Hoagland, M. Nastasi, B.P. Uberuaga, Science 327 (2010) $1631-1634$.

[2] K.Y. Yu, D. Bufford, C. Sun, Y. Liu, H. Wang, M.A. Kirk, M. Li, X. Zhang, Nat Commun 4 (2013) 1377.

[3] G.R. Odette, M.J. Alinger, B.D. Wirth, Annual Review of Materials Research 38 (2008) 471-503.

[4] W. Han, M.J. Demkowicz, N.A. Mara, E. Fu, S. Sinha, A.D. Rollett, Y. Wang, J.S. Carpenter, I.J. Beyerlein, A. Misra, Adv. Mater. 25 (2013) 6975-6979. 
[5] T.M. Robinson, M.L. Jenkins, Phil. Mag. A 43 (1981) 999-1015.

[6] L.M. Wang, R.A. Dodd, G.L. Kulcinski, J. Nucl. Mater. 155-157, Part 2 (1988) $1241-1248$.

[7] J.W. Yeh, S.K. Chen, S.J. Lin, J.Y. Gan, T.S. Chin, T.T. Shun, C.H. Tsau, S.Y. Chang, Adv. Engin. Mater. 6 (2004) 299-303.

[8] B. Cantor, I.T.H. Chang, P. Knight, A.J.B. Vincent, Mater. Sci. and Engin. A 375-377 (2004) 213-218.

[9] O.N. Senkov, G.B. Wilks, J.M. Scott, D.B. Miracle, Intermetallics 19 (2011) 698-706.

[10] B. Gludovatz, A. Hohenwarter, D. Catoor, E.H. Chang, E.P. George, R.O. Ritchie, Science 345 (2014) 1153-1158.

[11] J.W. Yeh, JOM 67 (2015) 2254-2261.

[12] Y. Zhang, G.M. Stocks, K. Jin, C. Lu, H. Bei, B.C. Sales, L. Wang, L.K. Beland, R.E. Stoller, G.D. Samolyuk, M. Caro, A. Caro, W.J. Weber, Nat Commun 6 (2015) 8736.

[13] D.S. Aidhy, C. Lu, K. Jin, H. Bei, Y. Zhang, L. Wang, W.J. Weber, Acta Mater. 99 (2015) 69-76.

[14] T. Nagase, S. Anada, P.D. Rack, J.H. Noh, H. Yasuda, H. Mori, T. Egami, Intermetallics 26 (2012) 122-130.

[15] T. Nagase, P.D. Rack, J.H. Noh, T. Egami, Intermetallics 59 (2015) 32-42.

[16] Z. Wu, Y.F. Gao, H. Bei, Scripta Mater. 109 (2015) 108-112.

[17] M.L. Jenkins, M.A. Kirk, Characterization of radiation damage by transmission electron microscopy, Institute of Physics Pub., Bristol, 2001. 
[18] O.S. Oen, Cross Sections for Atomic Displacements in Solids by Fast Electrons, Oak Ridge National Laboratory, Oak Ridge1965.

[19] A. Bourret, Phys. Stat. Sol. (a) 4 (1971) 813-825.

[20] D.J. Bacon, H.F. Deng, F. Gao, J. Nucl. Mater. 205 (1993) 84-91.

[21] F. Maury, M. Biget, P. Vajda, A. Lucasson, P. Lucasson, Phys. Rev. B 14 (1976) 5303-5313.

[22] N. Juslin, K. Nordlund, J. Wallenius, L. Malerba, Nucl. Instrum. Meth. B 255 (2007) $75-77$.

[23] L.M. Howe, Phil. Mag. 22 (1970) 965-981.

[24] K. Urban, Phys. Stat. Sol. (a) 4 (1971) 761-772.

[25] G. Das, T.E. Mitchell, J. Nucl. Mater. 56 (1975) 297-306.

[26] P. Czurratis, R. Keoggel, V. Syneček, M. Simerska, H. Loffler, Phys. Stat. Sol. (a) 106 (1988) 407-418.

[27] G. Thomas, J. Washburn, Reviews of Modern Physics 35 (1963) 992-1011.

[28] D. Kuhlmann-Wilsdorf, H.G.F. Wilsdorf, J. Appl. Phys. 31 (1960) 516-525.

[29] M.J. Makin, Phil. Mag. 20 (1969) 1133-1146.

[30] D.I.R. Norris, Phil. Mag. 22 (1970) 1273-1278. 

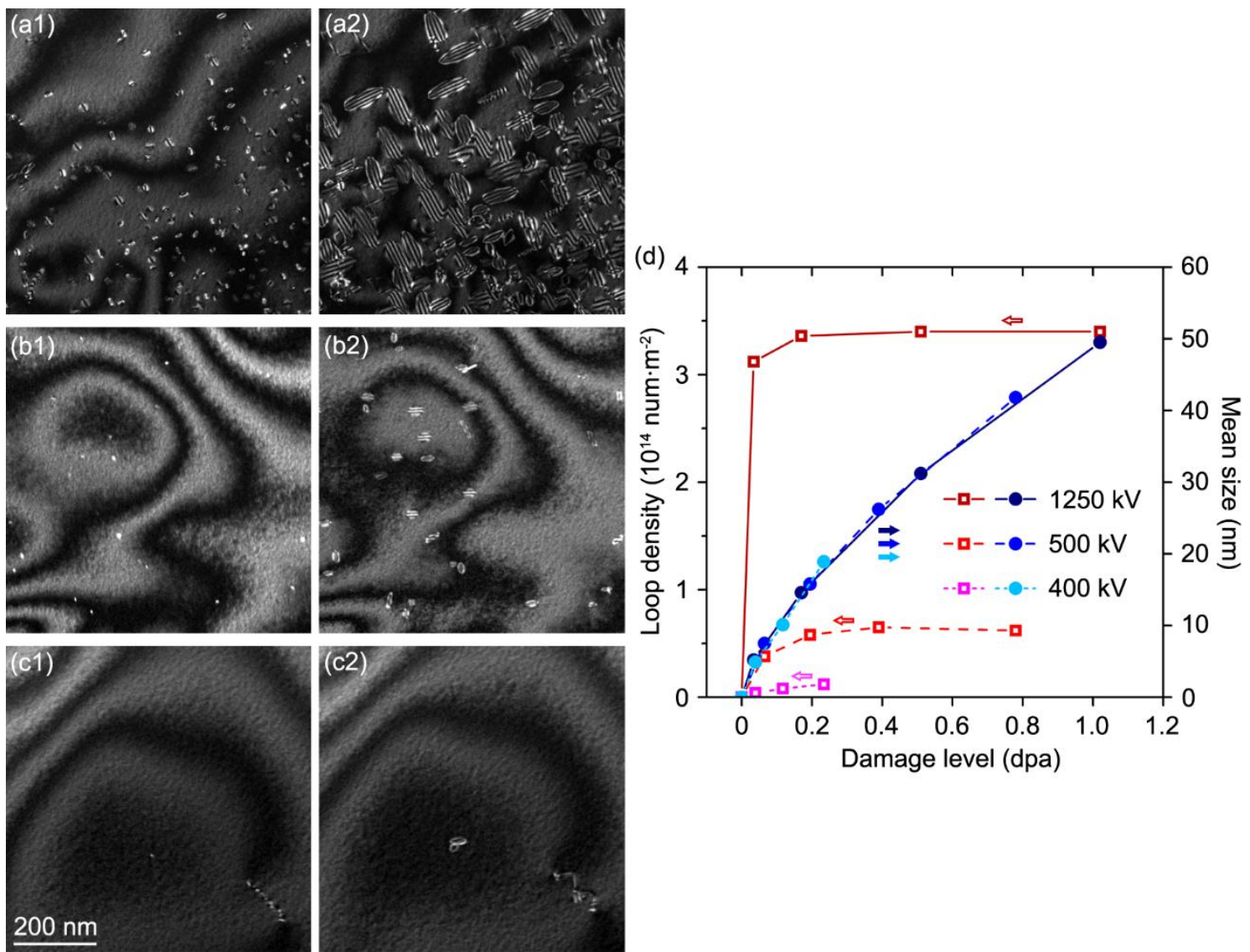

Figure 1. Defect production and growth in $\mathrm{CrFeCoNi}$ during in situ electron irradiation. (a-c) Representative microstructure as irradiated at $400{ }^{\circ} \mathrm{C}$ by (a) $1250 \mathrm{kV}$, (b) $500 \mathrm{kV}$, and (c) $400 \mathrm{kV}$ electron beam for (1) $5 \mathrm{~min}$ and (2) $30 \mathrm{~min}$, respectively. Images were recorded using (g, 3.1g) WBDF condition with $\mathbf{g}=(200)^{*}$. (d) Evolution of density (open squares) and mean size (solid circles) of loops with increasing damage level $\left(E_{\mathrm{d}}=15 \mathrm{eV}\right)$ at different beam energies $(1250 \mathrm{kV}$ : solid lines; $500 \mathrm{kV}$ : dash lines; $400 \mathrm{kV}$ : dot lines). 

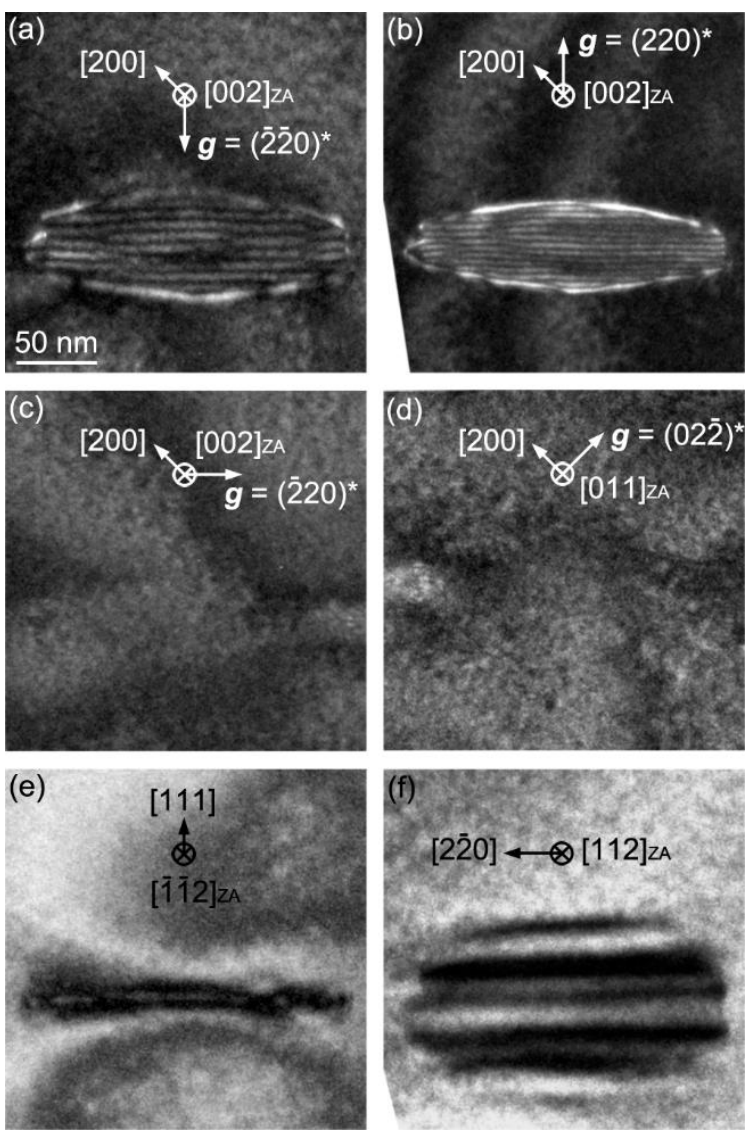

Figure 2. Identification of an interstitial-type, elliptical Frank loop in (111) plane with Burgers vector $\mathbf{b}=[\overline{1} \overline{1} \overline{1}] / 3$. (a-d): (g, 3.1g) WBDF images. (e, f) BF images. 

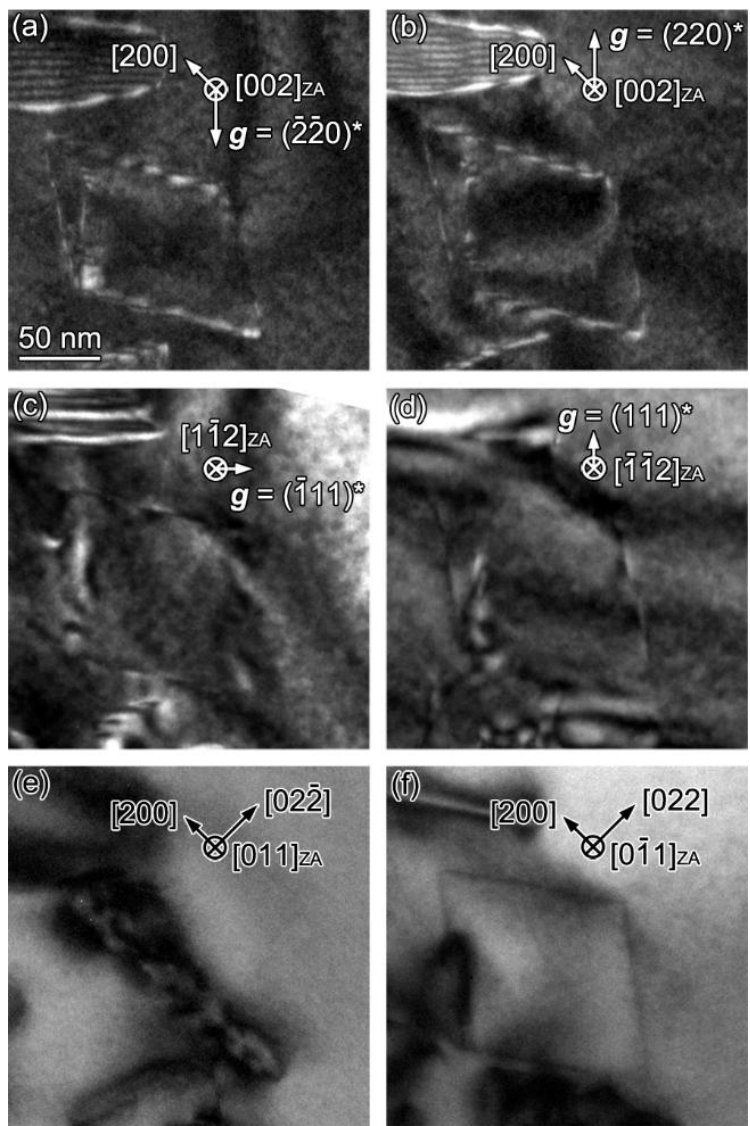

Figure 3. Identification of an interstitial-type, rhombus perfect loop in (01 $\overline{1})$ plane with Burgers vector $\mathbf{b}=[01 \overline{1}] / 2$ and edges $\mathbf{u}=[211]$ and [211]. (a-d): (g, 3.1g) WBDF images. (e, f) BF images. 

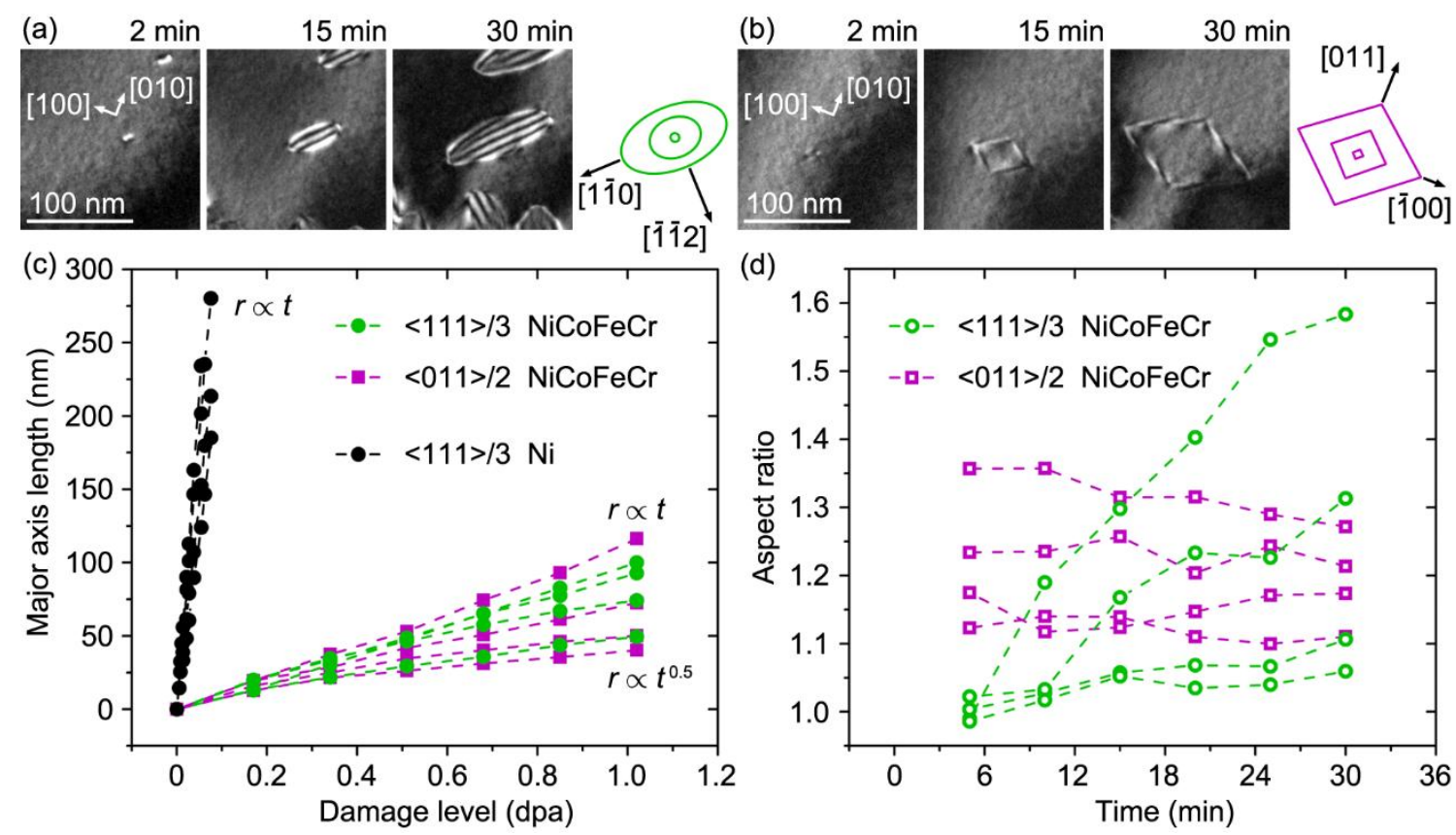

Figure 4. Growth behavior of individual loops in $\mathrm{CrFeCoNi}$ irradiated by $1250 \mathrm{kV}$ electron beam at $400{ }^{\circ} \mathrm{C}$. (a,b) Growth of (a) a Frank loop and (b) a perfect loop viewed in time-series of $(\mathbf{g}, 3.1 \mathbf{g})$ WBDF images with $\mathbf{g}=(200)^{*}$. Schematic: outlines of the loops viewed in their habit planes. (c) Increase of major axis length with damage level $\left(E_{\mathrm{d}}=15 \mathrm{eV}\right)$ measured in several Frank loops (green) and perfect loops (purple). Dash lines: fitting by power law. Black: Linear growth of Frank loops in pure $\mathrm{Ni}\left(E_{\mathrm{d}}=23 \mathrm{eV}\right)$ irradiated by $650 \mathrm{kV}$ electron beam at $420{ }^{\circ} \mathrm{C}$ [24]. (d) Evolution of aspect ratio with time measured in the same Frank loops (green) and perfect loops (purple) as in (c). 


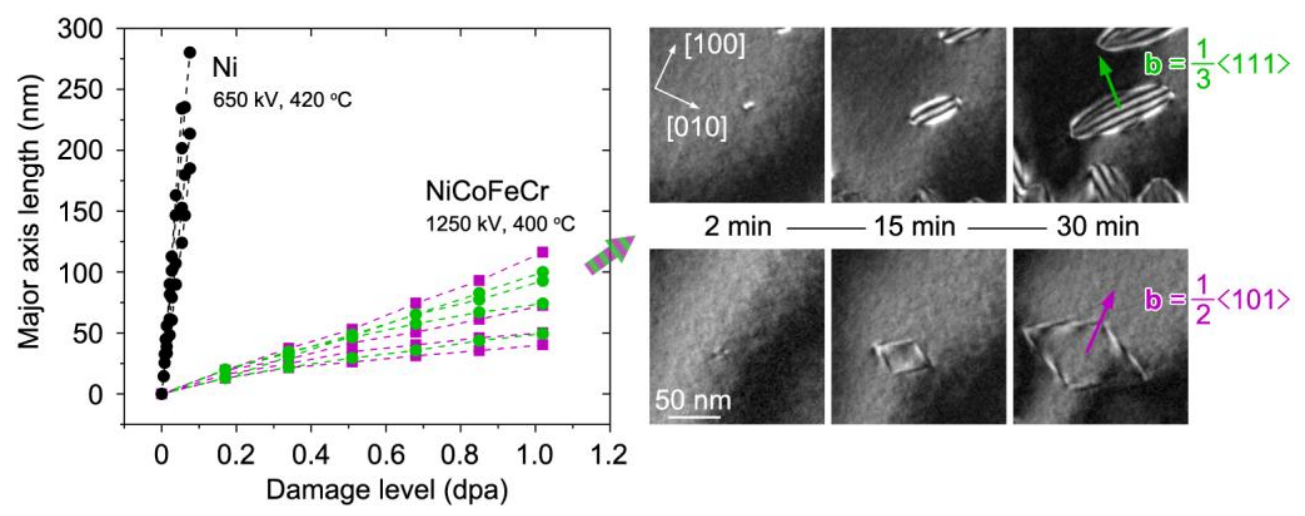

Graphical abstract 